Contents List available at RAZI Publishing

Matriks Sains Matematik (MSMK)

Journal Homepage: http://www.razipublishing.com/journals/matriks-sainsmatematik

https://doi.org/10.26480/msmk.01.2017.21.24

\title{
Study of Nonlocal Boundary Value Problems of Non-Integer Order Hybrid Differential Equations
}

\author{
Samina, Kamal Shah and Rahmat Ali Khan
}

${ }^{1}$ Department of Mathematics, University of Malakand, Dir(L), Khyber Pakhtunkhwa, Pakistan*Corresponding Author email: kamalshah408@gmail.com

This is an open access article distributed under the Creative Commons Attribution License, which permits unrestricted use, distribution, and reproduction in any medium, provided the original work is properly cited

\section{ARTICLE DETAILS}

Article history:

Received 30 September 2016

Accepted 15 December 2016

Available online 13 January 2017

\section{Keywords and phrases:}

Boundary value problems, Coupled system, Fractional differential equations, Existence and Uniqueness of solutions, Hybrid fixed point theorems. AMS Subject Classification: 34A08; 35R11

\section{ABSTRACT}

We study sufficient conditions for existence and uniqueness of solutions to boundary value problems (BVPs) for fractional hybrid differential equations(FHDEs) of the form

$$
\left\{\begin{array}{l}
\mathbf{D}^{\sigma}[\alpha(t)-\Phi(t, \alpha(t))]=\Psi\left(t, \beta(t), I^{\theta}(\beta(t))\right), t \in \mathrm{l}, \\
\mathbf{D}^{\sigma}[\beta(t)-\Phi(t, \beta(t))]=\Psi\left(t, \alpha(t), I^{\theta}(\alpha(t))\right), t \in \mathrm{l}, \\
\alpha(0)=0, \alpha(1)=\delta \alpha(\omega), \beta(0)=0, \beta(1)=\delta \beta(\omega),
\end{array}\right.
$$

where $\mathbf{I}=[0,1], \sigma, \theta \in(1,2]$ and $\delta, \omega \in(0,1)$. We use hybrid fixed point theorem due to Dhage and develop adequate results for existence of solutions to the proposed system of (FHDEs). We also provide a numerical problem to demonstrate our main results.

\section{INTRODUCTION}

Fractional calculus studied properties of fractional order integrals and derivatives. This area includes the notion and techniques for solving fractional order differential equations. Fractional calculus has recently treated into a hot topic for researchers in various scientific and engineering fields. The Systematic development is available in the books (Miller and Ross, 1993a; Baleanu et al., 2012a). Recently fractional order differential equations of non-integer order have attracted great consideration for their intensive applications in various field of science (Baleanu et al., 2012b; Samko et al., 1993a).

Perturbation methods or techniques are very much skillful for solving dynamical systems. Different types of perturbations of differential equations and their classification are available (Hilfer, 2000). Hybrid differential equations can be treated with hybrid fixed point theory (Herzallah and Baleanu, 2012a; Herzallah, 2012b; Kilbas et al., 2006a) Recently, a scientist developed sufficient condition for existence and uniqueness of solution to the following first order initial value problem for hybrid differential equation with quadratic perturbation (Dhage and Lakshmikantham, 2010).

$$
\begin{aligned}
& \frac{\mathbf{d}}{\mathbf{d t}}\left[\frac{\alpha(t)}{\Phi(t, \alpha(t))}\right]=\Psi(t, \alpha(t)), t \in \mathrm{I}, \\
& \alpha\left(t_{0}\right)=\alpha_{0} \in \mathbf{R},
\end{aligned}
$$

Further, they developed some essential differential inequalities and evaluation results. The results were further extended to the class of first order initial value problem for hybrid differential equation with linear perturbation of second type (Dhage and Jadhav, 2013).

$$
\begin{aligned}
& \frac{\mathbf{d}}{\mathbf{d t}}[\alpha(t)-\Phi(t, \alpha(t))]=\Psi(t, \alpha(t)), t \in \mathrm{I}, \\
& \alpha\left(t_{0}\right)=\alpha_{0} \in \mathbf{R} .
\end{aligned}
$$

Recently, extended the studies to hybrid differential equations of noninteger order, and they established appropriate conditions for existence of solutions to the following initial value problem for hybrid differential equations of non-integer order (Lu et al., 2013).

$$
\begin{aligned}
& \mathbf{D}^{\sigma}[\alpha(t)-\Phi(t, \alpha(t))]=\Psi(t, \alpha(t)), 0<\sigma<1, \text { a.e } t \in \mathrm{I}, \\
& \alpha\left(t_{0}\right)=\alpha_{0} \in \mathbf{R},
\end{aligned}
$$

where $\Phi, \Psi \in C(\mid \times \mathbf{R}, \mathbf{R})$. Recently, a researcher established enough conditions for existence of solutions to the following coupled system of initial value problem for hybrid differential equations of noninteger order (Bashiri et al., 2016).

$\mathbf{D}^{\sigma}[\alpha(t)-\Phi(t, \alpha(t))]=\Psi\left(t, \beta(t), I^{\theta}(\beta(t))\right), t \in \mathrm{l}, 0<\sigma \leq 1,0<\theta \leq 1$,

$\mathbf{D}^{\sigma}[\beta(t)-\Phi(t, \beta(t))]=\Psi\left(t, \alpha(t), I^{\theta}(\alpha(t))\right), t \in \mathrm{I}, 0<\sigma \leq 1,0<\theta \leq 1$, $\alpha(0)=0, \beta(0)=0$.

Motivated by the above studies, in this paper, we enlarge the outcomes to the case of non-local boundary value problems and investigate sufficient conditions for existence of solutions to the following system of three point boundary value problems for hybrid differential equations of non-integer order

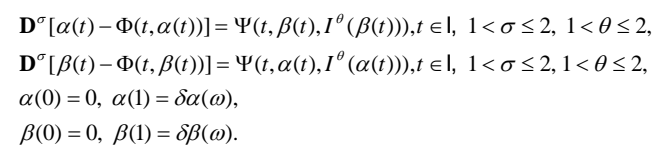

We use some classical tools of functional Analysis to develop appropriate conditions for existence of solutions. We also provide a numerical problem to show the applicability of our results.

\section{Preliminaries}

We denote the set of real numbers by $\mathbf{R}$ and bounded interval by I in R. Let $\Phi \in \mathbf{C}(\mid \times \mathbf{R} \times \mathbf{R}, \mathbf{R})$ and $\Psi \in C(\mid \times \mathbf{R} \times \mathbf{R}, \mathbf{R})$. Where $C(\mathrm{l} \times \mathbf{R} \times \mathbf{R}, \mathbf{R})$ denotes the class of continuous functions and $\mathbf{C}(I \times \mathbf{R} \times \mathbf{R}, \mathbf{R})$ denotes the class of functions called the Caratheodory class of function on $1 \times \mathbf{R} \times \mathbf{R}$ such that

(i) the map $t \rightarrow \Psi(t, \alpha, \beta)$ is measurable for each $\alpha, \beta \in \mathbf{R}$;

(ii) the map $\alpha \rightarrow \Psi(t, \alpha, \beta)$ is measurable for each $\alpha \in \mathbf{R}$ 
(iii) the map $\beta \rightarrow \Psi(t, \alpha, \beta)$ is continuous for each

$\beta \in \mathbf{R}$.

Definition 2.1 (Kilbas et al., 2006b) The Riemann-Liouville fractional derivative of order $\rho$ of a continuous function $\Phi:(a,+\infty) \rightarrow \mathbf{R}$ is defined as

$$
\mathbf{D}^{\rho} \Phi=\frac{1}{\Gamma(m-\rho)}\left(\frac{\mathbf{d}}{\mathbf{d t}}\right)^{m} \int_{a}^{t} \frac{\Phi(\vartheta)}{(t-\vartheta)^{\rho-m+1}} d \vartheta,
$$

where $m=[\rho]+1, m=[\rho]$ denotes the integral part of number $\rho$, provided that the right-hand side is point wise defined on $(a,+\infty)$.

Definition 2.2 (Kilbas et al., 2006b) The Caputo fractional order derivative of a function $\Phi$ on the interval $[a, b]$ is defined by

$$
{ }^{c} \mathbf{D}^{\rho} \Phi(t)=\frac{1}{\Gamma(m-\rho)} \int_{a}^{t}(t-\vartheta)^{m-\rho-1} \Phi^{(m)}(\vartheta) d \vartheta,
$$

where $m=[\rho]+1$ and $[\rho]$ represents the integer part of $\rho$

Note: Throughout this paper, we use Caputo fractional order derivative.

Definition 2.3 (Kilbas et al., 2006b) The Riemann-Liouville fractional integral of order $\rho$ of a function $\Phi:(0,+\infty) \rightarrow \mathbf{R}$ is

$$
I^{\rho} \Phi(t)=\frac{1}{\Gamma(\rho)} \int_{a}^{t}(t-\vartheta)^{\rho-1} \Phi(\vartheta) d \vartheta
$$

provided that the right-hand side is point wise defined on $(0,+\infty)$

Lemma 2.4 (Lakshmikantham et al., 2009) The following result holds for fractional differential equations

$$
I^{\rho} \mathbf{D}^{\rho} \alpha(t)=\alpha(t)+e_{0}+e_{1} t+e_{2} t^{2}+\ldots+e_{m-1} t^{m-1},
$$

for arbitrary $e_{i} \in \mathbf{R}, i=0,1,2, \ldots, m-1$.

Lemma 2.5 (Dhage, 2004) Let $C$ be a nonempty, closed convex and bounded subset of a Banach algebra $\mathbf{X}$ and let $S: \mathbf{x} \rightarrow \mathbf{x}$ and $T: C \rightarrow \mathrm{x}$ be two operators such that

(i) $S$ is nonlinear contraction;

(ii) $T$ is completely continuous;

(iii) $S x+T x \in C$ for all $x \in C$.

Then the operator equation $S x+T x=x$ has a solution in $C$.

Definition 2.6 (Chang et al., 1996) An element $(\alpha, \beta) \in \mathbf{X} \times \mathbf{x}$ is called a coupled fixed point of a mapping $A: \mathbf{x} \times \mathbf{x} \rightarrow \mathbf{X}$ if $A(\alpha, \beta)=\alpha$ and $A(\alpha, \beta)=\beta$.

Let $\phi: \mathbf{R}^{+} \rightarrow \mathbf{R}^{+}$denote the family of all functions fulfilling $\phi(n)<n$ for $n>0$ and $\phi(n)=0$. By a solution of FHDE, we mean a function $(\alpha, \beta) \in A C(\mathrm{l}, \mathbf{R} \times \mathbf{R})$ such that

(i) the function $t \rightarrow \alpha-\Phi(t, \alpha)$ is absolutely continuous for each $\alpha \in \mathbf{R}$; and

(ii) $(\alpha, \beta)$ satisfies the system of Equation in (1.2),

where $A C(\mathrm{l}, \mathbf{R} \times \mathbf{R})$ is the space of absolutely continuous real valued functions defined on I.

We place FHDE in $(1.2)$ the space $C(\mathrm{I}, \mathbf{R})$ of continuous real-valued functions defined on I. Define a supremum norm $\|\cdot\|$ in $C(\mathbf{l}, \mathbf{R})$ by $\|\alpha\|=\sup _{t \in 1}|\alpha(t)|$. Clearly, $X=C(\mathbf{l}, \mathbf{R})$ is a Banach algebra with respect to the above norm. The product space $\mathbf{x} \times \mathbf{X}$ is a vector space on $\mathbf{R}$ under the operations of addition and scalar multiplication and is a Banach space under the norm $\|(\alpha, \beta)\|=\|\alpha\|+\|\beta\|$.

The following result is useful for our main results.

Theorem 2.7 (Bashiri et al., 2016) Let $C$ be a nonempty, closed, convex and bounded subset of the Banach algebra $X$ and $\tilde{S}=C \times C$. Suppose that $T: \mathbf{x} \rightarrow \mathbf{x}$ and $S: \tilde{S} \rightarrow \mathbf{x}$ are two operators such that

( $\left.c_{1}\right)$ there exists $f_{\gamma}>0$ such that for all $x, y \in \mathbf{X}$, we have

$\|T(\alpha)-T(\beta)\| \leq v f_{\gamma}\|\alpha-\beta\|$, for $\quad$ some constant $v>0$;

(c2) $S$ is completely continuous,

(c $\left.c_{3}\right) \quad \alpha=T(\alpha)+S(\beta)$ for all $\beta \in C$ implies that $\alpha \in C$.

Then the operator $A(\alpha, \beta)=T(\alpha)+S(\beta)$ has at least a coupled fixed point in $\widetilde{S}$ whenever $v<1$.

\section{Existence results}

For the existence of solutions to the system (1.2) we introduce the supposition:

(do) the function $t \rightarrow \alpha-\Phi(t, \alpha)$ is increasing in $\mathbf{R}$ for all

$t \in \mathrm{I}$;

$\left(d_{1}\right) \quad\|\Phi(t, \alpha(t))-\Phi(t, \beta(t))\| \leq \eta\|\alpha-\beta\|$ where $\eta \leq \frac{1-\delta \omega}{(1-\delta \omega)+(1+\delta)} ;$

$\left(d_{2}\right) \quad$ Fix $\Phi_{0}=\max _{t \in I}|\phi(t, 0)|$;

$\left(d_{3}\right) \quad$ there exists $h \in C(J, \mathbf{R})$ such that

$\Psi(t, \alpha(t), \beta(t)) \leq h(t), \alpha, \beta \in \mathbf{R}, t \in \mathrm{I}$.

Lemma 3.1 Suppose that $\left(d_{0}\right)$ holds and $\Phi \in C(\mid \times \mathbf{R}, \mathbf{R})$ with $\Phi(0,0)=0$. Then for $\beta \in C(1, \mathbf{R})$ and $\theta \geq 0$, the unique solution of the boundary value problem

$\mathbf{D}^{\sigma}[\alpha(t)-\Phi(t, \alpha(t))]=\Psi\left(t, \beta(t), I^{\theta} \beta(t)\right), 1<\sigma, \theta \leq 2, t \in \mathrm{I}$

$$
\alpha(0)=0, \alpha(1)=\delta \alpha(\omega), \delta \omega<1
$$

is given by

$\alpha(t)=\Phi(t, \alpha(t))+\frac{t}{(1-\delta \omega)}[\delta \Phi(\omega, \alpha(\omega))-\Phi(1, \alpha(1))]+I^{\sigma} \Psi(t)+\frac{t}{(1-\delta \omega)}\left[\delta I^{\sigma} \Psi(\omega)-I^{\sigma} \Psi(1)\right]$

Let choose $\Phi_{0}=\max _{t \in \mid}|\Phi(t, 0)|$ and $N \geq\left(1+\frac{1+\delta}{1-\delta}\right)\left[L+\Phi_{0}+\frac{|k| t \mid}{\Gamma(\sigma+1)}\right]$. Define a subset $\tilde{S}$ of $X$ by $\tilde{S}=\{\alpha \in X:\|\alpha\| \leq N\}$. Now it is easy to show that $\tilde{S}$ is convex, bounded and closed subset of the Banach space $X$. Now we consider the system (1.2). In view of Lemma 3.1, $(\alpha(t), \beta(t))$ is a solution of the system (1.2) if and only if $(\alpha(t), \beta(t))$ satisfies the following system of integral equations

$$
\begin{aligned}
& \alpha(t)=\Phi(t, \alpha(t))+\frac{t}{(1-\delta \omega)}[\delta \Phi(\omega, \alpha(\omega))-\Phi(1, \alpha(1))]+I^{\sigma} \Psi(t)+\frac{t}{(1-\delta \omega)}\left[\delta I^{\sigma} \Psi(\omega)-I^{\sigma} \Psi(1)\right], \\
& \beta(t)=\Phi(t, \beta(t))+\frac{t}{(1-\delta \omega)}[\delta \Phi(\omega, \beta(\omega))-\Phi(1, \beta(1))]+I^{\sigma} \Psi(t)+\frac{t}{(1-\delta \omega)}\left[\delta I^{\sigma} \Psi(\omega)-I^{\sigma} \Psi(1)\right],
\end{aligned}
$$

(3.1)

where $t \in \mathrm{I}$. Define two operators $T: X \rightarrow X$ and $S: \tilde{S} \rightarrow X$ by

$$
\begin{aligned}
& T \alpha(t)=\Phi(t, \alpha(t))+\frac{t}{(1-\delta \omega)}[\delta \Phi(\omega, \alpha(\omega))-\Phi(1, \alpha(1))] \\
& S \alpha(t)=I^{\sigma} \Psi(t)+\frac{t}{(1-\delta \omega)}\left[\delta I^{\sigma} \Psi(\omega)-I^{\sigma} \Psi(1)\right]
\end{aligned}
$$

then the system of integral equations (3.1) modified into the system of operator equations

$$
\begin{aligned}
& \alpha(t)=T \alpha(t)+S \beta(t) \\
& \beta(t)=T \beta(t)+S \alpha(t), t \in \mathrm{I},
\end{aligned}
$$

and solutions of the system (3.1) are fixed points of the system (3.3). Lemma 3.2 By the hypothesis $\left(d_{1}\right)$, the operator $T$ defined by (3.2) is contraction.

Proof. For $\alpha, \beta \in X$, we have

$$
\begin{aligned}
|T \alpha(t)-T \beta(t)| & =\mid \Phi(t, \alpha(t))+\frac{t}{(1-\delta \omega)}[\delta \Phi(\omega, \alpha(\omega))-\Phi(1, \alpha(1))] \\
& -\Phi(t, \beta(t))-\frac{t}{(1-\delta \omega)}[\delta \Phi(\omega, \beta(\omega))-\Phi(1, \beta(1))] \mid \\
& \leq|\Phi(t, \alpha(t))-\Phi(t, \beta(t))|+\frac{\delta t}{(1-\delta \omega)}|\Phi(\omega, \alpha(\omega))-\Phi(\omega, \beta(\omega))| \\
& +\frac{t}{(1-\delta \omega)}|\Phi(1, \alpha(1))-\Phi(1, \beta(1))| \\
& \leq\left(1+\frac{\delta}{(1-\delta \omega)}+\frac{1}{(1-\delta \omega)}\right)|\Phi(t, \alpha(t))-\Phi(t, \beta(t))|
\end{aligned}
$$

which in view of the assumption $\left(d_{1}\right)$ implies that $|T \alpha-T \beta| \leq\left(1+\frac{\delta+1}{(1-\delta \omega)}\right) \eta\|\alpha-\beta\|$. Hence it follows that $\|T \alpha-T \beta\| \leq\left(1+\frac{\delta+1}{(1-\delta \omega)}\right) \eta\|\alpha-\beta\|$. This means that $T$ is contraction on $X$. 
Lemma 3.3 Under the assumption $\left(d_{2}\right)$, the operator $S$ defined by (3.2) is continuous and compact.

Proof. For continuity of $S$, choose a sequence $\alpha_{m} \in \tilde{S}$ which converges to a point $\alpha$ in $\widetilde{S}$. Then, by dominated convergence theorem, we have

$$
\begin{aligned}
\lim _{m \rightarrow \infty} S \alpha_{m}(t) & =\frac{1}{\Gamma(\sigma)} \int_{0}^{t}(t-\vartheta)^{\sigma-1} \lim _{m \rightarrow \infty} \Psi\left(\vartheta, \alpha_{m}(\vartheta), I^{\theta}\left(\alpha_{m}(\vartheta)\right)\right) d \vartheta \\
& +\frac{t \delta}{(1-\delta \omega) \Gamma(\sigma)} \int_{0}^{\omega}(\omega-\vartheta)^{\sigma-1} \lim _{m \rightarrow \infty} \Psi\left(\vartheta, \alpha_{m}(\vartheta), I^{\theta}\left(\alpha_{m}(\vartheta)\right)\right) d \vartheta \\
& -\frac{t}{(1-\delta \omega) \Gamma(\sigma)} \int_{0}^{1}(1-\vartheta)^{\sigma-1} \lim _{m \rightarrow \infty} \Psi\left(\vartheta, \alpha_{m}(\vartheta), I^{\theta}\left(\alpha_{m}(\vartheta)\right)\right) d \vartheta
\end{aligned}
$$

Using continuity of $\Psi$, it follows that

$$
\begin{aligned}
\lim _{m \rightarrow \infty} S \alpha_{m}(t) & =\frac{1}{\Gamma(\sigma)} \int_{0}^{t}(t-\vartheta)^{\sigma-1} \Psi\left(\vartheta, \alpha(\vartheta), I^{\theta}(\alpha(\vartheta))\right) d \vartheta \\
& +\frac{t \delta}{(1-\delta \omega) \Gamma(\sigma)} \int_{0}^{\omega}(\omega-\vartheta)^{\sigma-1} \Psi\left(\vartheta, \alpha(\vartheta), I^{\theta}(\alpha(\vartheta))\right) d \vartheta \\
& -\frac{t}{(1-\delta \omega) \Gamma(\sigma)} \int_{0}^{1}(1-\vartheta)^{\sigma-1} \Psi\left(\vartheta, \alpha(\vartheta), I^{\theta}(\alpha(\vartheta))\right) d \vartheta=S \alpha(t), t \in \mathrm{I} .
\end{aligned}
$$

Hence, $S$ is continuous on $\tilde{S}$.

For boundedness of the operator $S$ on $\tilde{S}$, choose $\alpha \in \tilde{S}$ and using hypothesis $\left(d_{2}\right)$, we have

$$
\begin{aligned}
|S \alpha(t)| & \leq \frac{1}{\Gamma(\sigma)}\left|\int_{0}^{t}(t-\vartheta)^{\sigma-1} \Psi\left(\vartheta, \alpha(\vartheta), I^{\theta}(\alpha(\vartheta))\right) d \vartheta\right| \\
& +\frac{t \delta}{(1-\delta \omega) \Gamma(\sigma)}\left|\int_{0}^{\omega}(\omega-\vartheta)^{\sigma-1} \Psi\left(\vartheta, \alpha(\vartheta), I^{\theta}(\alpha(\vartheta))\right) d \vartheta\right| \\
& +\frac{t}{(1-\delta \omega) \Gamma(\sigma)}\left|\int_{0}^{1}(1-\vartheta)^{\sigma-1} \Psi\left(\vartheta, \alpha(\vartheta), I^{\theta}(\alpha(\vartheta))\right)\right| d \vartheta \\
& \leq \frac{1}{\Gamma(\sigma)}\left[\int_{0}^{t}(t-\vartheta)^{\sigma-1}|h(\vartheta)| d \vartheta\right. \\
& \left.+\frac{t \delta}{(1-\delta \omega)} \int_{0}^{\omega}(\omega-\vartheta)^{\sigma-1}|h(\vartheta)| d \vartheta+\frac{t}{(1-\delta \omega)} \int_{0}^{1}(1-\vartheta)^{\sigma-1}|h(\vartheta)|\right] d \vartheta .
\end{aligned}
$$

Hence, it follows that

$$
\|S \alpha\| \leq \frac{1}{\Gamma(\sigma+1)}\left(1+\frac{\delta}{(1-\delta \omega)}+\frac{1}{(1-\delta \omega)}\right)\|h\|, \text { for all } \alpha \in S,
$$

which implies that $S$ is uniformly bounded on $B$. For equid-continuity of $S$, choose $t_{1}, t_{2} \in \mathrm{I}$ such that $t_{1}<t_{2}$ and $\alpha \in B$, then, we have

$$
\begin{aligned}
\left|S \alpha\left(t_{1}\right)-S \alpha\left(t_{2}\right)\right| & \leq \frac{1}{\Gamma(\sigma)} \mid \int_{0}^{t_{1}}\left(t_{1}-\vartheta\right)^{\sigma-1} \Psi\left(\vartheta, \alpha(\vartheta), I^{\theta}(\alpha(\vartheta))\right) d \vartheta \\
& +\frac{t_{1} \delta}{1-\delta \omega} \int_{0}^{\omega}(\omega-\vartheta)^{\sigma-1} \Psi\left(\vartheta, \alpha(\vartheta), I^{\theta}(\alpha(\vartheta))\right) d \vartheta \\
& -\frac{t_{1}}{1-\delta \omega} \int_{0}^{1}(1-\vartheta)^{\sigma-1} \Psi\left(\vartheta, \alpha(\vartheta), I^{\theta}(\alpha(\vartheta))\right) d \vartheta \\
& -\int_{0}^{t_{2}}\left(t_{2}-\vartheta\right)^{\sigma-1} \Psi\left(\vartheta, \alpha(\vartheta), I^{\theta}(\alpha(\vartheta))\right) d \vartheta \\
& -\frac{t_{2} \delta}{1-\delta \omega} \int_{0}^{\omega}(\omega-\vartheta)^{\sigma-1} \Psi\left(\vartheta, \alpha(\vartheta), I^{\theta}(\alpha(\vartheta))\right) d \vartheta \\
& +\frac{t_{2}}{1-\delta \omega} \int_{0}^{1}(1-\vartheta)^{\sigma-1} \Psi\left(\vartheta, \alpha(\vartheta), I^{\theta}(\alpha(\vartheta))\right) d \vartheta \mid .
\end{aligned}
$$

Which implies that

$$
\begin{aligned}
\left|S \alpha\left(t_{1}\right)-S \alpha\left(t_{2}\right)\right| \leq & \frac{1}{\Gamma(\sigma)}\left|\int_{0}^{f_{0}^{\prime}}\left(\left(t_{1}-\vartheta\right)^{\sigma-1}-\left(t_{2}-\vartheta\right)^{\sigma-1}\right) \Psi\left(\vartheta, \alpha(\vartheta), I^{\sigma}(\alpha(\vartheta))\right) d \vartheta\right| \\
& +\frac{\delta}{1-\delta \omega}\left|\int_{a}^{\omega}\left(t_{1}-t_{2}\right)(\omega-\vartheta)^{\sigma-1} \Psi\left(\vartheta, \alpha(\vartheta), I^{\sigma}(\alpha(\vartheta))\right) d \vartheta\right| \\
& +\frac{1}{1-\delta \omega}\left|\int_{0}^{1}\left(t_{1}-t_{2}\right)(1-\vartheta)^{\sigma-1} \Psi\left(\vartheta, \alpha(\vartheta), I^{\sigma}(\alpha(\vartheta))\right) d \vartheta\right| \\
& \left.\frac{\|h\|}{\Gamma(\sigma+1)} \mid\left(t_{1}\right)^{\sigma}-\left(t_{2}\right)^{\sigma}\right)\left|+\frac{\| h \mid \delta}{(1-\delta \omega) \Gamma(\sigma+1)}\right|\left|\left(t_{1}-t_{2}\right)\right| \\
& +\frac{\|h\|}{(1-\delta \omega) \Gamma(\sigma+1)}\left|\left(t_{1}-t_{2}\right)\right| \\
& \left.=\frac{\|h\|}{\Gamma(\sigma+1)}\left[\mid\left(t_{1}\right)^{\sigma}-\left(t_{2}\right)^{\sigma}\right)\left|+\frac{1+\delta}{(1-\delta \omega)}\right|\left(t_{1}-t_{2}\right) \mid\right] \\
& \leq \frac{\|h\|}{\Gamma(\sigma+1)}\left[\sigma\left|\left(t_{1}-t_{2}\right)\right|+\frac{1+\delta}{(1-\delta \omega)}\left|\left(t_{1}-t_{2}\right)\right|\right] \\
& =\frac{\|h\|}{\Gamma(\sigma+1)}\left(\sigma+\frac{1+\delta}{(1-\delta \omega)}\right)\left|\left(t_{1}-t_{2}\right)\right| \rightarrow 0 \text { as } t_{1} \rightarrow t_{2},
\end{aligned}
$$

which shows that $S$ is equip-continuous. Hence, $S$ is completely continuous on $\tilde{S}$.

Theorem 3.4 Assume that the hypothesis $\left(d_{0}\right)-\left(d_{3}\right)$ hold, Then the of system has a solution on I.

Proof. It is enough to show that system (3.3) has a fixed point. In view of Lemma 3.2, the operator $T$ has a contraction. In view of lemma 3.3, the operator $S$ is continuous and compact.

Now, for $\alpha \in X$ and $\beta \in \tilde{S}$ such that $\alpha=T \alpha+S \beta$, we have

$$
\begin{aligned}
|\alpha(t)| \leq|T \alpha(t)|+|S \beta(t)| & \left|\Phi(t, \alpha(t))+\frac{t}{(1-\delta \omega)}[\delta \Phi(\omega, \alpha(\omega))-\Phi(1, \alpha(1))]\right| \\
& +\frac{1}{\Gamma(\sigma)} \mid \int_{0}^{t}(t-\vartheta)^{\sigma-1} \Psi\left(\vartheta, \beta(\vartheta), I^{\theta}(\beta(\vartheta))\right) d \vartheta \\
& +\frac{t \delta}{1-\delta \omega} \int_{0}^{\omega}(\omega-\vartheta)^{\sigma-1} \Psi\left(\vartheta, \beta(\vartheta), I^{\theta}(\beta(\vartheta))\right) d \vartheta \\
& -\frac{t}{1-\delta \omega} \int_{0}^{1}(1-\vartheta)^{\sigma-1} \Psi\left(\vartheta, \beta(\vartheta), I^{\theta}(\beta(\vartheta))\right) d \vartheta \mid \\
& \leq|\Phi(t, \alpha(t))|+\frac{\delta}{1-\delta \omega}|\Phi(\omega, \alpha(\omega))|+\frac{1}{1-\delta \omega}|\Phi(1, \alpha(1))| \\
& +\frac{1}{\Gamma(\sigma)}|h|\left[\frac{t^{\sigma}}{\sigma}+\frac{\delta \omega^{\sigma}}{(1-\delta \omega) \sigma}+\frac{1}{(1-\delta \omega) \sigma}\right] \\
& \leq\left(1+\frac{\delta}{1-\delta \omega}+\frac{1}{1-\delta \omega}\right)|\Phi(t, \alpha(t))|+\frac{1}{\Gamma(\sigma+1)} \mid h \|\left(\left(1+\frac{\delta}{1-\delta \omega}+\frac{1}{1-\delta \omega}\right)\right. \\
& =\left(1+\frac{\delta}{1-\delta \zeta}+\frac{1}{1-\delta \omega}\right)\left[|\Phi(t, \alpha(t))-\Phi(t, 0)|+|\Phi(t, 0)|+\frac{\|h\|}{\Gamma(\sigma+1)}\right] \\
& \leq\left(1+\frac{\delta}{1-\delta \omega}+\frac{1}{1-\delta \omega}\right)\left[L+\Phi_{0}+\frac{\|h\|}{\Gamma(\sigma+1)}\right] \\
& =\left(1+\frac{1+\delta}{1-\delta \omega}\right)\left[L+\Phi{ }_{0}+\frac{\|h\|}{\Gamma(\sigma+1)}\right] \leq N .
\end{aligned}
$$

which implies that $\beta \in \tilde{S}$. Hence by Theorem 2.7, the system (1.2) has a coupled fixed point in $\tilde{S}$.

Example 3.5 Consider the following coupled system of fractional hybrid differential equation with boundary conditions.

$$
\left\{\begin{array}{l}
{ }^{c} \mathbf{D}^{\frac{3}{2}}\left[\alpha(t)-\left(e^{-t}+\frac{\frac{\left(t^{2}+1\right)}{8}|\alpha(t)|}{20+|\alpha(t)|}\right)\right]=\frac{t^{2}}{2}-\frac{\left(t^{3}+1\right)}{20}\left[\sin |\beta(t)|+\cos \left|I^{\frac{3}{2}} \beta(t)\right|\right], t \in[0,1], \\
{ }^{c} \mathbf{D}^{\frac{3}{2}}\left[\beta(t)-\left(e^{-t}+\frac{\frac{\left(t^{2}+1\right)}{8}|\beta(t)|}{20+|\beta(t)|}\right)\right]=\frac{t^{2}}{2}-\frac{\left(t^{3}+1\right)}{20}\left[\sin |\alpha(t)|+\cos \left|I^{\frac{3}{2}} \alpha(t)\right|\right], t \in[0,1], \\
\alpha(0)=0, \alpha(1)=\frac{1}{2} \alpha\left(\frac{1}{2}\right), \\
\beta(0)=0, \beta(1)=\frac{1}{2} \beta\left(\frac{1}{2}\right) .
\end{array}\right.
$$

From (3.4), we have

$$
\begin{aligned}
& \Phi(t, \alpha(t))=e^{-t}+\frac{\left(t^{2}+1\right)}{30} \frac{\frac{(t+1)}{4}|\alpha(t)|}{20+|\alpha(t)|} \\
& \Psi(t, \alpha(t), \beta(t))=\frac{t^{2}}{2}-\frac{\left(t^{3}+1\right)}{20}\left[\sin |\beta(t)|+\sin \left|I^{\frac{3}{2}} \beta(t)\right|\right] \leq \frac{t^{2}}{2} .
\end{aligned}
$$

Now it is easy to find $L=\frac{1}{4}, M=20, \Phi_{0}=1,\|h\|=\frac{1}{6}, \sigma=\frac{3}{2}$.

$$
\left(1+\frac{1+\delta}{1-\delta \omega}\right)\left[L+\Phi_{0}+\frac{\|h\|}{\Gamma(\sigma+1)}\right]=3\left(\frac{5}{4}+\frac{2}{9 \sqrt{\pi}}\right)=12.37<13
$$

Hence all the conditions of Theorem 3.4, are satisfied, which show that the FHDEs system (3.4) has a solution in $\tilde{S}=\{x \in X:\|x\| \leq 13\}$. 


\section{References}

[1] Baleanu, D., Diethelm, K., Scalas, E., Trujillo, J.J., (2012a) Fractional Calculus Models and Numerical Methods. Nonlinearity and Chaos, World Scientific, Boston, Mass, USA, 3.

[2] Baleanu, D., Diethelm, K., Scalas, E., Trujillo, J. J., (2012b) Fractional Calculus Models and Numerical Methods, Series on Complexity, Nonlinearity and Chaos 3, World Scientific.

[3] Bashiri, T., Vaezpour, S.M., Park, C., (2016). A coupled fixed point theorem and application to fractional hybrid differential problems. Fixed Point Theory and Applications,1-12.

[4] Cattani, C., (2012). Fractional calculus and Shannon wavelet, Mathematical Problems in Engineering, 26 pages. Chang, S.S., Cho, Y.J., Huang, N.J., (1996). Coupled fixed point theorems with applications.

[5] Journal of the Korean Mathematical Society, 33(3): 575-585. Dhage, B.C., Jadhav, N.S., (2013). Basic result in the theory of hybrid differential equation with linear perturbation of second type. Tamkang Journal of Mathematics. 44(2):171-186.

[6] Dhage, B.C., (2004). A fixed point theorem in Z anach algebras with applications to functional integral equations. Kyungpook Mathematical Journal, 44(10-11):145-155.

[7] Dhage, Z.C., Lakshmikantham, V., (2010). Z basic results on hybrid differential equations, Nonlinear Analysis, 4(3): 414-424.

[8] Herzallah, M.A.E., Baleanu, D., (2012a). Existence of a periodic mild solution for a nonlinear fractional differential equation. Computers and Mathematics with Applications, 64(10): 3059-3064, Herzallah, M.A.E., El-Sayed, A.M.A., Baleanu, D., (2010). On the fractional-order diffusionwave process. Romanian Journal in Physics, 55(3-4): 274-284. Herzallah M.A.E., El-Shahed, M., Baleanu, D., (2013). Mild and strong solutions for a fractional nonlinear Neumann boundary value problem. Journal of Computational Analysis

and Applications, 15(2): 341-352.

[9] Herzallah, M.A.E., (2012b). Mild and strong solutions to few types of fractional order nonlinear equations with periodic boundary conditions. Indian Journal of Pure and Applied Mathematics, 43(6) 619-635.

[10] Hilfer, R., (2000). Applications of Fractional Calculus in Physics, World Scientific, New Jersey,

NJ, USA.

[11] Kilbas, A. A., Srivastava, H. M., and Trujillo, J. J., (2006a). Theory and Applications of Fractional Differential Equations. North-Holland Mathematical Studies, Elsevier, Amsterdam, The

Netherlands, 204.

[12] Kilbas, A.A., Srivastava, H.M., Trujillo, J.J., (2006b). Theory and Applications of Fractional DifferentialEquations. Elsevier, Amsterdam. Lakshmikantham, V., Leela, S., Vasundhara, J., (2009). Theory of Fractional Dynamic Systems. Cambridge Academic Publishers, Cambridge, UK.

[13] Lu, H., Sun, S., Yang, D., Teng, H., (2013). Theory of fractional hybrid differential equations with linear perturbations of second type. Boundary Value Problems. 2013, 1-23.

[14] Miller, K.S., Ross, B., (1993a). An Introduction to the Fractional Calculus and Fractional Differential Equations, John Wiley and Sons, New York, NY, USA, Miller, K.S., Ross, Z., (1993b). An Introduction to the Fractional Calculus and Differential Equations, A Wiley-Inter-Science Publication. John Wiley and Sons, NewYork, NY, USA.

[15] Podlubny, I., (1999a). Fractional Differential Equations. Mathematics in Science and Engineering, Academic Press, San Diego, Calif, USA, 198.

[16] Podlubny, (1999b). Fractional Differential Equations
Academic Press, New York, NY, USA.

[17] Samko, S.G., Kilbas, A.A., Marichev, O.I., (1993a). Fractional Integrals and Derivatives: Theory and Applications. Gordon and Breach, New York, NY, USA. Samko, S.G., Kilbas, A.A., Marichev, O.I., (1993b). Fractional Integrals and Derivatives, Gordon and Breach Science Publishers, Yverdon, Switzerland. 